\title{
Enjeux cardiaques chez les patients atteints de COVID-19
}

\author{
Oscar Calvillo-Argüelles MD, Heather J. Ross MD MHSc
}

Citation : CMAJ 2020 June 8;192:E630. doi : 10.1503/cmaj.200562-f; diffusion hâtive le $1^{\text {er }}$ mai 2020

Voir la version anglaise de l'article ici : www.cmaj.ca/lookup/doi/10.1503/cmaj.200562

1

On observe des lésions cardiaques chez environ le quart des patients atteints de maladie à coronavirus 2019 (COVID-19) hospitalisés

De $8 \%$ à $28 \%$ des patients hospitalisés présentent des lésions myocardiques, des taux de troponines qui dépassent le $99^{e}$ centile de la limite supérieure de la normale (LSN), résultat d'une atteinte myocardique indirecte (p. ex., hypoxémie, septicémie ou libération de cytokines) ou directe ${ }^{1,2}$. Les taux sont plus élevés chez les patients des unités de soins intensiff ${ }^{1,2}$. Une élévation progressive des troponines et des peptides natriurétiques a été observée et semble proportionnelle à l'intensité de la maladie, avec des pics qui sont atteints 2 à 3 semaines après le déclenchement des symptômes (annexe 1, disponible en anglais au www.cmaj.ca/lookup/suppl/doi:10.1503/cmaj.200562/-/DC1)2,3.

Les biomarqueurs cardiaques sont utiles à la stratification du risque

Chez les patients hospitalisés, la vérification périodique des taux de troponines et de peptides natriurétiques permet de cibler les patients exposés à un risque accru de dysfonction ventriculaire gauche ou d'arythmie. Si les taux sont élevés, envisager un suivi plus étroit des patients et une échographie cardiaque au lieu d'intervention en cas d'élévation modérée (> 3 fois la LSN) ou d'instabilité hémodynamique et/ou électrique².

L'infarctus du myocarde (IM) peut survenir sans coronaropathie obstructive Les patients peuvent présenter un IM causé par un apport en oxygène insuffisant par rapport à la demande ou par une thrombose microvasculaire. La myocardite peut prendre les allures d'un syndrome coronarien aigu; les patients sont alors exposés à un risque accru de choc cardiogénique ${ }^{2}$. Pour les patients qui présentent des douleurs rétrosternales réfractaires ou récurrentes, une élévation modérée des taux de troponines ou des anomalies électrocardiographiques focalisées, envisager une échographie cardiaque au lieu d'intervention pour vérifier s'il y a présence de dysfonction ventriculaire gauche, et une coronarographie par tomodensitométrie pour écarter un diagnostic de coronaropathie ${ }^{2,4}$.

4 Le traitement doit cibler les causes spécifiques de l'atteinte myocardique

Traiter l'insuffisance cardiaque, l'IM avec sus-décalage du segment ST et la dysfonction ventriculaire gauche chez un patient atteint de COVID-19 conformément aux protocoles établis en revêtant l'équipement de protection individuelle approprié, y compris les traitements médicaux préconisés par les lignes directrices. Toute investigation hémodynamique invasive se limitera aux patients dont il est impossible d'évaluer le statut volémique sur une base clinique ${ }^{2}$. On dispose de peu de données sur la prise en charge de la myocardite fulminante et le cas échéant, les décisions thérapeutiques devraient être prises par des équipes multidisciplinaires ${ }^{1,2}$. Les patients qui prennent des inhibiteurs de l'enzyme de conversion de l'angiotensine ${ }^{1,2}$ et des bloqueurs des récepteurs de l'angiotensine II ne doivent pas cesser leurs traitements ${ }^{5}$.

5 Les complications cardiaques sont associées à un pronostic sombre Jusqu'à $40 \%$ des décès associés à la COVID-19 pourraient, du moins en partie, être attribués à l'insuffisance cardiaque ${ }^{2}$. Chez les patients hospitalisés qui développent une insuffisance cardiaque, les taux de mortalité pourraient atteindre $60 \%{ }^{3}$. On observe de la tachycardie ou de la fibrillation ventriculaire soutenue chez jusqu'à $17 \%$ des patients qui présentent une atteinte cardiaque et elles sont plus fréquentes chez les patients de soins intensifs ${ }^{2}$.

\section{Références}

1. Driggin E, Madhavan MV, Bikdeli B, et al. Cardiovascular considerations for patients, health care workers, and health systems during the coronavirus disease 2019 (COVID-19) pandemic. J Am Coll Cardiol 2020 le 19 mars [Cyberpublication avant impression]. pii: S0735-1097(20)34637-4. doi: 10.1016/j.jacc.2020.03.031.

2. ESC guidance for the diagnosis and management of CV disease during the COVID-19 pandemic. Sophia Antipolis (France): European Society of Cardiology; mis à jour 2020 le 21 avril. Accessible ici : www.escardio.org/Education/COVID-19-and -Cardiology/ESC-COVID-19-Guidance (consulté le 25 avril 2020).

3. Zhou F, Yu T, Du R, et al. Clinical course and risk factors for mortality of adult inpatients with COVID-19 in Wuhan, China: a retrospective cohort study. Lancet 2020;395:1054-62.

4. Bangalore S, Sharma A, Slotwiner A, et al. STsegment elevation in patients with COVID-19: a case series. N Engl J Med 2020 le 17 avril [Cyberpublication avant impression]. doi: 10.1056/ NEJMc2009020.

5. Bavishi C, Maddox TM, Messerli FH. Coronavirus disease 2019 (COVID-19) infection and renin angiotensin system blockers. JAMA Cardiol 2020 le 3 avril [Cyberpublication avant impression]. doi: 10.1001/jamacardio.2020.1282.

Intérêts concurrents : Aucun déclaré Cet article a été révisé par des pairs.

Affiliation : Division de cardiologie, Département de médecine, Centre de cardiologie Peter Munk, Réseau universitaire de santé, Toronto, Ont.

Correspondance : Heather Ross, Heather.ross@uhn.ca 